\title{
1D-Var retrieval of daytime total columnar water vapour from MERIS measurements
}

\author{
R. Lindstrot ${ }^{1}$, R. Preusker ${ }^{1}$, H. Diedrich ${ }^{1}$, L. Doppler ${ }^{1}$, R. Bennartz ${ }^{2}$, and J. Fischer ${ }^{1}$ \\ ${ }^{1}$ Institut für Weltraumwissenschaften, Freie Universität Berlin, Carl-Heinrich-Becker-Weg 6-10, 12165 Berlin, Germany \\ ${ }^{2}$ Department of Atmospheric and Oceanic Sciences, University of Wisconsin-Madison, 1225 W. Dayton Street, \\ Madison, WI, USA
}

Correspondence to: R. Lindstrot (rasmus.lindstrot@wew.fu-berlin.de)

Received: 30 September 2011 - Published in Atmos. Meas. Tech. Discuss.: 8 November 2011

Revised: 9 March 2012 - Accepted: 12 March 2012 - Published: 27 March 2012

\begin{abstract}
A new scheme for the retrieval of total columnar water vapour from measurements of MERIS (Medium Resolution Imaging Spectrometer) on ENVISAT (ENVIronmental SATellite) is presented. The algorithm is based on a fast forward model of the water vapour transmittance around $900 \mathrm{~nm}$, including a correction for atmospheric scattering and the influence of the temperature- and pressure-profile on the water vapour absorption lines. It provides the water vapour column amount for cloud-free scenes above land and ocean at a spatial resolution of $0.25 \mathrm{~km} \times 0.3 \mathrm{~km}$ and $1 \mathrm{~km} \times 1.2 \mathrm{~km}$, depending on whether applied to the "full resolution" or the operational "reduced resolution" mode of MERIS. Uncertainties are provided on a pixel-by-pixel basis, taking into account all relevant sources of error. An extensive validation against various sources of ground-based reference data reveals a high accuracy of MERIS water vapour above land (root mean square deviations between $1.4 \mathrm{~mm}$ and $3.7 \mathrm{~mm}$ ), apart from a wet bias of MERIS between 5 and $10 \%$ that is found in all comparison studies. This wet bias might be caused by spectroscopic uncertainties, such as the description of the water vapour continuum. Above ocean the accuracy is reduced, due to the uncertainty introduced by the unknown atmospheric scattering. Consequently, an increased root mean square deviation of $\geq 5 \mathrm{~mm}$ was found by comparing MERIS total columnar water vapour above ocean against SSM/I and ENVISAT MWR data. An increased wet bias of $2-3 \mathrm{~mm}$ is found over ocean, potentially due to a not properly working atmospheric correction scheme.
\end{abstract}

\section{Introduction}

\subsection{Background}

Due to the tremendous importance of water vapour for the greenhouse effect and the energy balance of the earth, the space-borne monitoring of water vapour column amounts and vertical profiles has a long history in remote sensing. As the water molecule absorbs electromagnetic radiation ranging from microwave to visible wavelengths, a number of different methods have been developed during the past decades, either making use of either active (e.g. Heise et al., 2006) or passive microwave measurements (e.g. SSM/I; Schlüssel and Emery, 1990) or mid- and long-wavelength infrared spectral range (e.g. AIRS; Susskind et al., 2003 or IASI; Pougatchev et al., 2009) or based on sensing the absorption of water vapour in the visible (e.g. GOME; Noël et al., 2002 or SCIAMACHY; Noël et al., 2004) or near infrared spectral range from MERIS or MODIS (e.g. Bennartz and Fischer, 2001; Gao and Kaufman, 2003; Albert et al., 2005; Guanter et al., 2008). Above ocean, microwave and infrared techniques have proven to be superior, due to the well known emissivity and temperature of the ocean surface. Over land, the water vapour signal at these wavelengths is blurred by the uncertain emissivity of the surface, limiting its main applicability to the sensing of upper-air humidity by using strongly absorbing bands. In the visible and near infrared spectral regions, the situation is reversed as the brighter land surfaces allow water vapour retrievals with a higher accuracy as compared 
to dark water surfaces, where the signal strongly depends on the uncertain influence of scattering in the atmosphere. The $\rho \sigma \tau$-absorption band between $0.9 \mu \mathrm{m}$ and $1 \mu \mathrm{m}$ is particularly well suited for water vapour sensing, due to the fact that all land surface types are bright at this spectral range. A number of spectrometers employed currently and in future, such as the Moderate Resolution Imaging Spectroradiometer (MODIS) or the Medium Resolution Imaging Spectrometer (MERIS) and their follow up models, provide measurements in spectral bands within and close-by this water vapour absorption band, allowing for the exploitation of the differential absorption.

\subsection{The Medium Resolution Imaging Spectrometer (MERIS)}

MERIS is a programmable, medium-spectral resolution, imaging spectrometer (Rast et al., 1999). It is one of ten core instruments on the polar orbiter ENVISAT (Environmental Satellite, launched on 1 March 2002) flying at $800 \mathrm{~km}$ in a sun-synchronous orbit with an equator crossing time of 10:00 a.m., descending node, and $98.5^{\circ}$ inclination. MERIS consists of 5 identical push-broom imaging spectrometers operating in the solar spectral range (390 to $1040 \mathrm{~nm}$ ), arranged in a fan shape configuration which covers a total field of view of $68.5^{\circ}$ and spans a swath width of around $1150 \mathrm{~km}$. The spectral dispersion is achieved by mapping the entrance slit of a grating spectrometer onto a CCD array. The integration time, instrument optics and CCD array resolution are adjusted such that MERIS has a spatial resolution of $260 \mathrm{~m} \times 300 \mathrm{~m}$ and a spectral sampling of $1.25 \mathrm{~nm}$. The instrument electronic data rate provides 15 channels which are programmable by ground command in width and in position. In the regular operation mode the spatial resolution is reduced by a factor of 4 along and across track ("reduced resolution" mode). In the "full resolution" mode, the full spatial resolution is transmitted. The central wavelengths of the spectral channels as listed in Table 1 vary slightly across the field of view of MERIS. This "spectral smile" is caused by curvature of the image of the slit formed in the focal plane array, resulting in viewing angle-dependent central wavelengths of the spectral MERIS channels.

\section{Physical background of the retrieval method}

The absorption of electromagnetic radiation by water vapour, occurring at characteristic wavelengths throughout the solar and terrestrial spectrum, is related to the excitation of various combinations of the three fundamental vibrational modes of the water molecule. Measurements of the reflected sun light within the resulting absorption bands enable a determination of the total columnar water vapour (hereafter referred to as TCWV), provided that
Table 1. Central wavelength and bandwidth (fwhm) of MERIS spectral channels.

\begin{tabular}{llll}
\hline Band & $\begin{array}{l}\text { Center } \\
(\mathrm{nm})\end{array}$ & $\begin{array}{l}\text { Width } \\
(\mathrm{nm})\end{array}$ & Usage \\
\hline 1 & 412.5 & 10 & Yellow substance, turbidity \\
2 & 442.5 & 10 & Chlorophyll \\
3 & 490 & 10 & Chlorophyll, pigment \\
4 & 510 & 10 & Suspended matter, turbidity \\
5 & 560 & 10 & Chlorophyll, suspended matter \\
6 & 620 & 10 & Suspended matter \\
7 & 665 & 10 & Chlorophyll \\
8 & 681.25 & 7.5 & Chlorophyll \\
9 & 708.25 & 10 & Atmospheric correction, "red edge" \\
10 & 753.75 & 7.5 & Cloud optical thickness, cloud-top \\
& & & pressure reference \\
11 & 761.875 & 3.75 & Cloud-top pressure \\
12 & 778 & 10 & Aerosol, vegetation \\
13 & 865 & 20 & Aerosol, atmospheric correction \\
14 & 885 & 10 & Water vapour reference \\
15 & 900 & 10 & Water vapour \\
\hline
\end{tabular}

1. incoming solar radiation is available, precluding nighttime retrievals,

2. the channel used is located in a non-saturated and therefore sufficiently sensitive part of the absorption band,

3. the optical thickness of other atmospheric species is either zero or precisely known,

4. the surface albedo is known or can be accurately estimated,

5. the paths of the detected photons through the atmosphere are either known to a sufficient degree or predominantly attributable to the unscattered, surfacereflected fraction,

6. the lower troposphere, holding the main part of TCWV, is not obscured by clouds or optically thick aerosol layers.

For monochromatic radiation, the transmittance $T$ through a medium can be related to its optical depth $\tau$ and the air mass $\mu$, following the Beer-Lambert law:

$T=\exp \left(-\frac{\tau}{\mu}\right)$.

Neglecting scattering processes and assuming non-saturated absorption, the mass of the absorbing species can thus be directly related to the logarithm of the traversed medium's transmittance. Since the transmittance cannot be measured directly from space, it is approximated by the ratio of two neighbouring bands with differing optical depth. Fischer (1988) proposed to use two closely spaced bands, with one 
channel located in the shortwave end of the $\rho \sigma \tau$-absorption band around $900 \mathrm{~nm}$ and the other one in the atmospheric window region around $885 \mathrm{~nm}$. At this spectral range, almost all land surfaces such as vegetated, bare soil or snow-covered areas, are bright and therefore provide a good background for retrievals of total columnar amounts of water vapour. The small spectral distance of merely $15 \mathrm{~nm}$ minimizes the difference in surface reflectance and atmospheric scattering properties between the two bands. Finally, by locating the absorption channel at $900 \mathrm{~nm}$ instead of sensing higher optical depths of water vapour at e.g. $950 \mathrm{~nm}$, it is assured that the measurements are sensitive to TCWV throughout the range of globally occurring water vapour column amounts. This is due to the fact that the water vapour absorption at $900 \mathrm{~nm}$ does not get saturated, even in case of high air masses in a humid atmosphere. Therefore, the MERIS bands (see Table 1) are perfectly suited for the daytime, cloud-free retrieval of TCWV over land.

The individual water vapour absorption lines are subject to pressure- and temperature-dependent broadening processes in the atmosphere. In the lower atmosphere, the dominant process is pressure broadening. The resulting line can approximately be described by the Lorentz line shape:

$f\left(v-v_{0}\right)=\alpha_{\mathrm{L}} / \pi /\left(\left(v-v_{0}\right)^{2}+\alpha_{\mathrm{L}}^{2}\right)$

where $\alpha_{\mathrm{L}}$ is the Lorentz half-width at half maximum, which is roughly proportional to the number of collisions per unit time and therefore a function of temperature and pressure:

$\alpha_{\mathrm{L}} \propto p T^{-1 / 2}$.

Figure 1 shows the global distribution of residual errors caused by deviations in the assumed temperature profile, calculated from GFS (Global Forecast System) data for one exemplary day. By analyzing the global distribution of temperature profiles, interpolated to 10:00 a.m. LT (local time) at every location on the globe, the bias of the assumed average temperature in the layer holding $90 \%$ of the humidity was calculated. If the actual surface temperature is used to estimate the temperature profile by mixing two standard profiles from McClatchey et al. (1972) (left panels), the errors in the average temperature and the resulting deviations in the derived TCWC are significantly reduced, as compared to a retrieval based on a fixed US standard profile (right panels). The residual TCWV error caused by the temperature profile uncertainty is reduced from $0.6 \mathrm{~mm}$ to $0.3 \mathrm{~mm}$ (root mean square error). The global bias of $0.6 \mathrm{~mm}$ is eliminated and systematic latitude-dependent biases are avoided.

Figure 2 shows the hypothetical effect of not accounting for the surface elevation and the resulting reduced surface pressure on the retrieval of TCWV, again based on globally analyzing a single day of GFS data. If a constant surface pressure of $1013 \mathrm{hPa}$ is assumed, the resulting deviation in the calculated transmittance causes relative biases of TCWV of more than $40 \%$ for elevated areas and dry conditions, such as found e.g. over the the Tibetan plateau. Over land, an average bias of $0.5 \mathrm{~mm}$ and a root mean square error of $0.9 \mathrm{~mm}$ of TCWV are avoided by accounting for the surface pressure effect on the absorption line width.

A further important factor for the retrieval of TCWV is the influence of scattering at particles and molecules in the atmosphere, which is especially important for measurements over dark surfaces. By introducing a scattering correction factor $f$, the influence of atmospheric scattering on the measured band ratio can be accounted for: $f$ represents the relation between the band ratio $R_{\text {noscat }}$ in case of pure absorption, i.e. assuming a non-scattering atmosphere, and the true band ratio $R$ :

$f=\frac{R}{R_{\text {noscat }}}$.

$f$ is larger than one, as atmospheric scattering through reflection of incoming solar radiation altogether causes a shortening of the average photon path length in the atmosphere and reduces the amount of water vapour along the photon path, by preventing a fraction of photons from traversing the humid boundary layer. Figure 3 shows $f$, calculated with radiative transfer simulations using the Matrix Operator Model (MOMO, Fischer and Grassl, 1984; Fell and Fischer, 2001; Hollstein and Fischer, 2012), and the equivalent error in retrieved TCWV when scattering is neglected as a function of Lambertian surface albedo and aerosol optical thickness at $900 \mathrm{~nm}$ for a total columnar water vapour of $56 \mathrm{~mm}$, a viewing zenith angle (VZA) of $30^{\circ}$, a solar zenith angle (SZA) of $64^{\circ}$ and a relative azimuth angle (RAA) of $0^{\circ}$ (三 sensor is placed opposite of sun). In the upper panels, $f$ was calculated for a continental aerosol located in the boundary layer, the lower panels correspond to an aerosol layer in the upper troposphere. Several conclusions can be drawn from the shown plots:

1. The scattering correction factor is close to 1 for bright surfaces because the measurement is dominated by photons that have been reflected at the bottom of the atmosphere, resulting in a small difference between $R$ and $R_{\text {noscat }}$.

2. Above dark surfaces such as the ocean, $f$ is increased, as the predominant part of the detected photons is reflected by atmospheric scatterers and thus does not traverse the whole vertical column of water vapour.

3. Correspondingly, there is a strong bias in retrieved TCWV if scattering is neglected above dark surfaces and a less pronounced effect for bright surfaces (see right panels).

4. Elevated aerosol layers have a much stronger effect on the measured transmittance as compared to scatterers located in the boundary layer (see lower panels). 

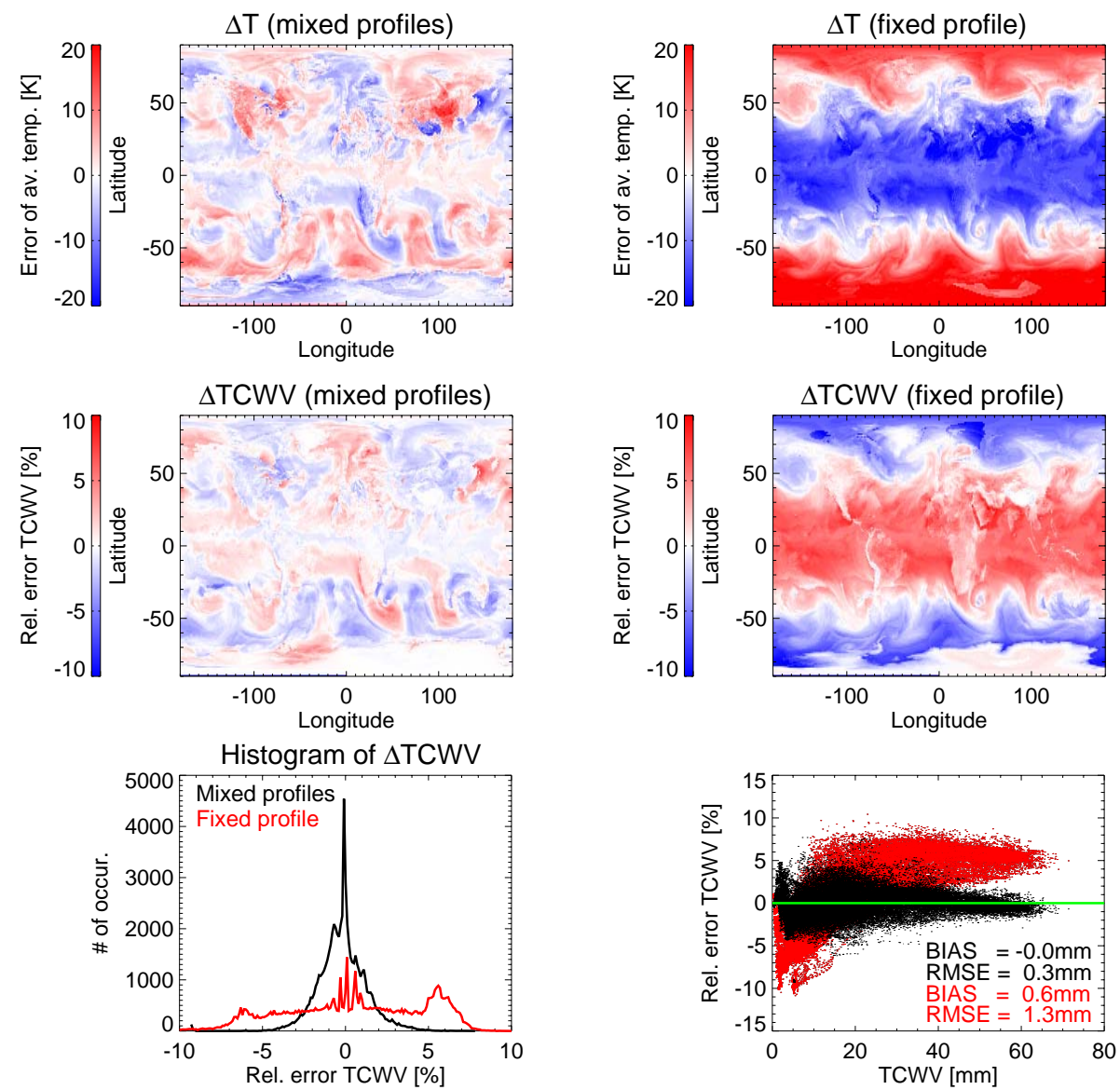

Fig. 1. Effect of temperature profile on TCWV retrieval: top panels show deviation of assumed average temperature in humid layer from the truth, middle panels show the resulting deviation of derived TCWV. Left panels represent the case of parametrizing the temperature profile with the surface temperature and mixing standard profiles accordingly. The right panels represent the assumption of a globally constant temperature profile (US standard). Bottom panels show global histograms of relative error and its dependency on TCWV, including both land and ocean pixels.

One of the most important parameters determining $f$ is thus the surface albedo. Figure 4 shows global histograms of land surface albedo at $900 \mathrm{~nm}$ throughout the year 2004, as extracted from the MERIS Albedomap (Muller et al., 2007), providing 16-day averages of surface albedo in the MERIS bands on a $0.05^{\circ} \times 0.05^{\circ}$ longitude-latitude grid. In the near infrared spectral region the land surface reflectance is commonly larger than 0.2. In case of boundary layer aerosols, this corresponds to a bias of $5-15 \%$ in TCWV if scattering is neglected. For a dark ocean surface, the bias is in the range of $20-30 \%$. In the less common case of highly elevated aerosols, these values are significantly higher.

Figure 5 shows the angular dependence of $f$ for fixed values of aerosol optical thickness $(\mathrm{AOT}=0.28)$ and surface albedo (0.4). The main statement here is, that the effect of atmospheric scattering on the measured band ratio is more pronounced for high zenith angles and there is an additional influence of the relative azimuth distance between sensor and sun. It is therefore mandatory to include the observation geometry with sufficient angular resolution in the calculation of $f$.

\section{1D-Var retrieval algorithm}

The current, operational algorithm for the retrieval of total columnar water vapour from MERIS measurements is based on a non-linear regression (Bennartz and Fischer, 2001; Fischer et al., 2010). The main shortcomings of this approach are

1. the lack of flexibility regarding the consideration of different boundary conditions such as the aerosol loading, the temperature profile or the surface pressure,

2. the lack of uncertainty estimates on a pixel-by-pixel basis.

In this work, an inverse modelling scheme is used for the retrieval of the water vapour column, therewith enabling both 

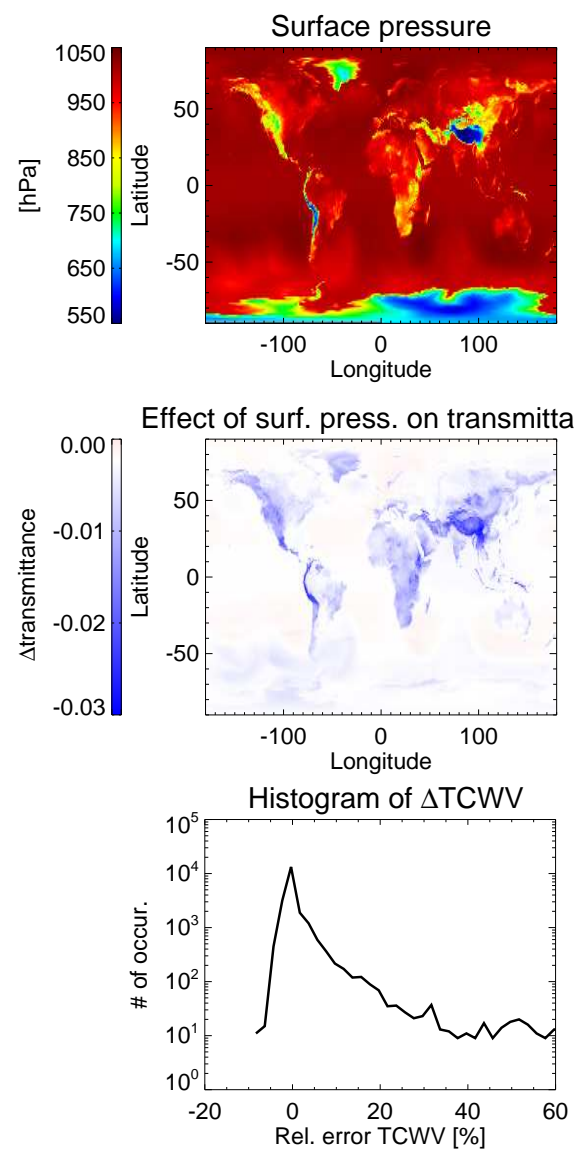
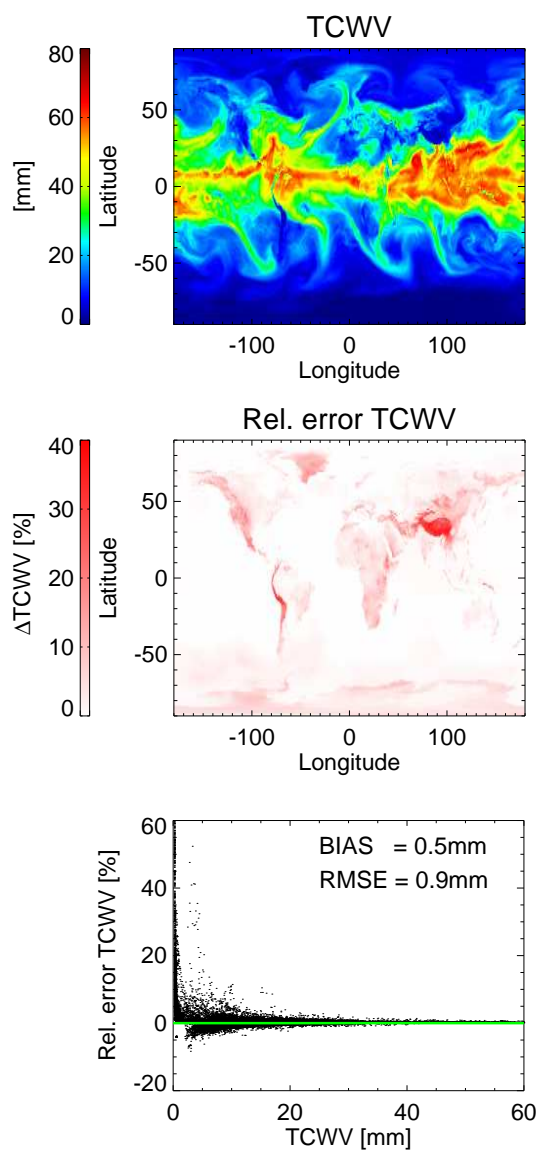

Fig. 2. Effect of surface pressure on TCWV retrieval: Top panels show surface pressure and TCWV on 2 June 2007 , taken from GFS reanalysis. Middle panels show impact on transmittance and resulting relative error of derived TCWV, based on the assumption of a constant surface pressure of $1013 \mathrm{hPa}$ (for constant solar zenith angle of $45^{\circ}$ and nadir view). Bottom panels show global histogram of relative error and its dependency on TCWV, shown only for land pixels.

the calculation of uncertainties and the inclusion of background knowledge as defined above. The drawback of this solution is a considerable increase in computing time, necessitating a fast forward simulation module.

The retrieval scheme is divided into several individual steps, starting with a determination of the surface reflectance in MERIS bands 14 and 15 and a subsequent iterative optimization of TCWV by minimizing the cost function $F$, represented by the absolute difference between the modeled and observed ratios of MERIS bands 15 and 14 : $F=\left|R_{\text {modelled }}-R_{\text {measured }}\right|$. In order to account for the influence of scattering on the measured water vapour transmittance, a scattering correction factor is calculated at each retrieval step, depending on the aerosol loading, surface reflectance, viewing geometry and TCWV itself. The uncertainty of the retrieved value of TCWV is calculated after the final iteration step, by taking into account uncertainties introduced by instrumental effects such as sensor noise and uncertainties in prior knowledge of the influencing parameters such as surface albedo, aerosol optical depth, aerosol scale height, temperature profile and surface pressure. The individual retrieval steps are detailed in the following sections.

\subsection{Forward model}

As detailed in the previous section, in the near infrared spectral range, the remotely sensable information about TCWV is represented by an approximation of the water vapour transmittance along the light path by a band ratio. In case of MERIS, bands 14 and 15 are used, located close-by and within the water vapour absorption band. However, since band 14 is affected by some isolated water vapour absorption lines and due to the influence of atmospheric scattering and the spectral distance of MERIS bands 14 and 15, mainly noticeable through differences in surface reflectance and solar incoming irradiance, the measured ratio of the radiances $L_{\mathrm{i}}, R=\frac{L_{15}}{L_{14}}$, deviates from the actual water vapour transmittance. The deviation is a function of the surface albedo and its spectral dependency, the viewing and illumination geometry and the vertical distribution and optical properties of the 

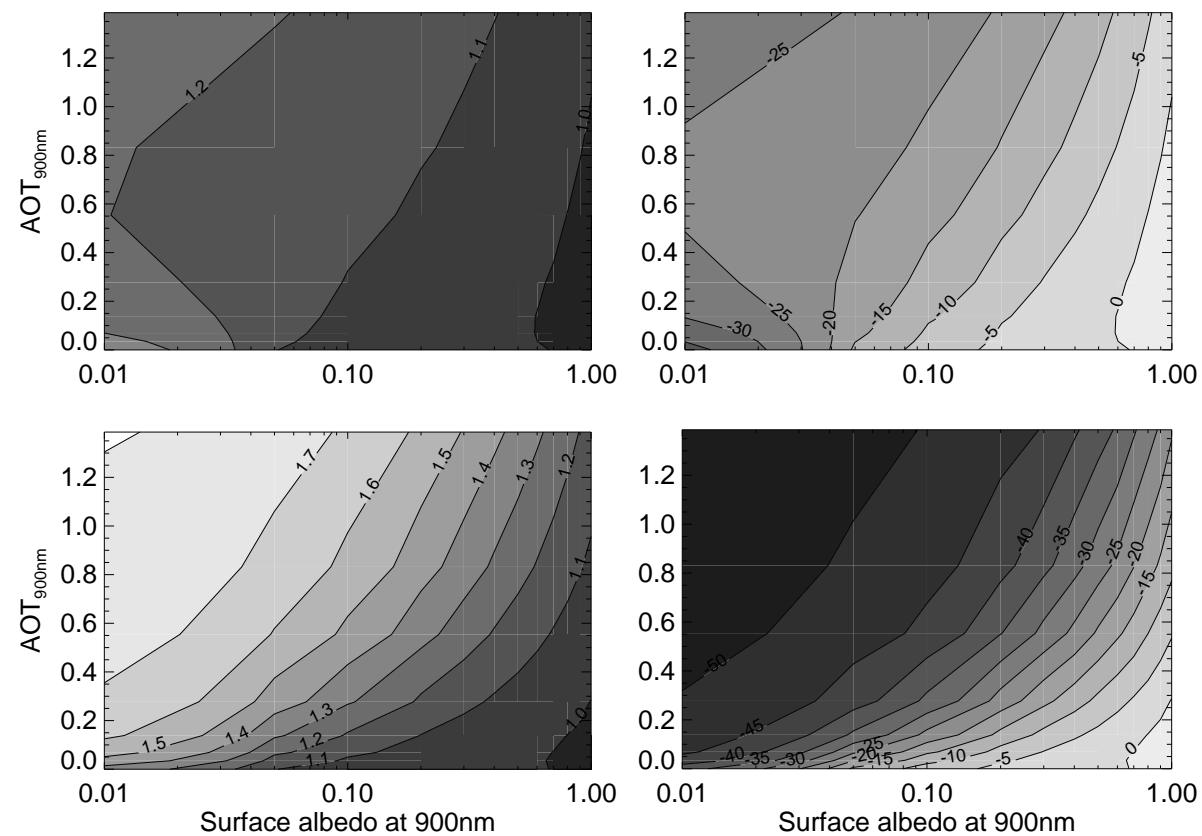

Fig. 3. Scattering correction factor $f$ as a function of surface albedo and aerosol optical thickness at $900 \mathrm{~nm}$ (left panels) and equivalent underestimation of TCWV in mm when scattering is neglected (right panels), shown for $\mathrm{SZA}=64^{\circ}, \mathrm{VZA}=30^{\circ}, \mathrm{RAA}=0^{\circ}, \mathrm{TCWV}=56 \mathrm{~mm}$ and a continental aerosol located in the boundary layer (upper panels) and the upper troposphere (lower panels), respectively.

atmospheric scatterers. In a first step, the measured band ratio $R$ is therefore normalized by the ratio of the solar incoming irradiance $E_{\mathrm{i}}$ (Thuillier et al., 2003) at the particular wavelengths of both channels: $R_{N}=\frac{L_{15}}{L_{14}} \cdot \frac{E_{14}}{E_{15}}$, taking into account the spectral smile effect, causing the central wavelength of MERIS bands to depend slightly on the viewing angle (e.g. Delwart et al., 2007; Lindstrot et al., 2010). Afterwards, $R_{N}$ is simulated by the ratio of the water vapour transmittance in both bands, corrected for the spectral surface albedo difference and scattering processes in the atmosphere:

A fast forward model of the normalized band ratio $R_{N}$ as a function of TCWV, the viewing geometry, and the surface albedo, temperature and pressure is needed for the optimization of TCWV. It uses stored absorption coefficients that were calculated from the HITRAN2008 line database (Rothman et al., 2009), using the AER LBLRTM code (Clough et al., 2005) and an advanced k-distribution method (Bennartz and Fischer, 2000), based on re-sorting the large number of quasi-monochromatic spectral intervals within each MERIS band with respect to their optical depth and combining them to a significantly lower number of pseudo-spectral intervals. The absorption coefficients were calculated for a fixed water vapour column amount $\left(\mathrm{WV}_{0}\right)$. The optical depths in each pseudo-spectral interval $i$ for a value of $\mathrm{WV}^{*}$ are then obtained from simply multiplying the stored coefficients accordingly: $\tau_{i}^{*}=\tau_{i 0} \frac{\mathrm{WV}^{*}}{\mathrm{WV}_{0}}$. In detail, for a given observing geometry and TCWV, the transmittance $T$ in each MERIS band is modeled by
1. calculating the optical depth $\tau_{i j}$ for each pseudospectral interval $i$ and vertical layer $j$ for the desired value of TCWV,

2. calculating the transmittance in each pseudo-spectral interval from the sum of the optical depth along the line of sight following Eq. (1) and

3. subsequently summing up all transmittance values with respect to the weights $w_{i}$ associated to the pseudospectral intervals:

$$
T=\sum_{i=1}^{\# \text { \#intervals }} w_{i} \cdot \exp \left(-\sum_{j=1}^{\text {\#layers }} \tau_{i j} / \mu\right) .
$$

The optical depth values $\tau_{i j}$ are stored in look-up tables for 6 standard temperature profiles (McClatchey et al., 1972) and 27 different pressure levels. The transmittance is calculated for the four look-up table grid points closest to the actual surface pressure and temperature of the considered scene, hereby assuming that the surface temperature is highly correlated with the actual vertical temperature profile (see Fig. 1). The actual surface pressure is derived from converting land elevation to pressure, using the GTOPO30 digital elevation model (US GeologicalSurvey, 1996), while the surface temperature is extracted from NWP reanalysis data. The final transmittances of MERIS bands 14 and 15 are then calculated as a weighted average among these four figures, with the weights determined by the distances to the closest grid points of temperature and pressure. 


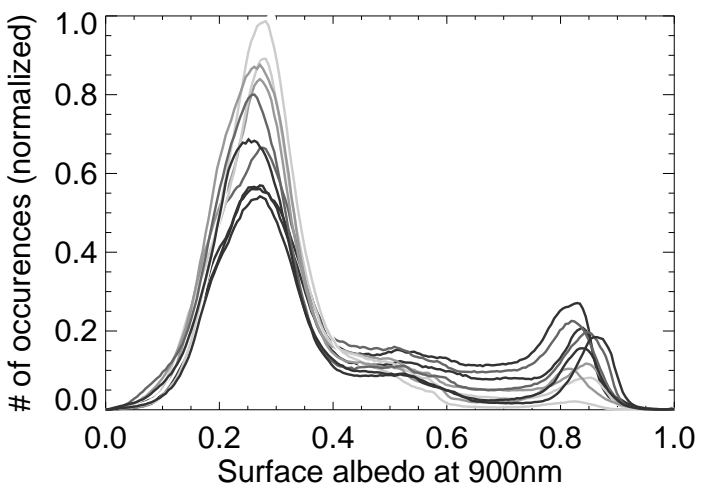

Fig. 4. Global, normalized histograms of surface albedo at $900 \mathrm{~nm}$ through the year 2004 (grey shading indicating seasonal dependence with dark grey indicating winter and light grey indicating summer months). The data was extracted from the MERIS Albedomap data set (Muller et al., 2007).

Following the calculation of the transmittances from the stored absorption coefficients, the ratio $R$ of both transmittances is modified with the difference in spectral surface reflectance $\rho_{\lambda}$ between the MERIS bands: $R^{*}=T_{900 \mathrm{~nm}} / T_{885 \mathrm{~nm}} \cdot \rho_{900 \mathrm{~nm}} / \rho_{885 \mathrm{~nm}}$. As the true surface reflectance at the MERIS wavelengths is unknown, it is estimated by correcting the measured reflectance at the top of the atmosphere (TOA) for the scattering component, using a look-up table approach for the atmospheric correction. As this procedure is possible only in window channels, the surface reflectance at $900 \mathrm{~nm}$ is linearly extrapolated from the reflectance in the window regions, $\rho_{865 \mathrm{~nm}}$ and $\rho_{885 \mathrm{~nm}}$. The atmospheric correction relies on assumptions about the aerosol loading, type and vertical distribution. However, over the relatively bright land surfaces the influence on the surface reflectance retrieval is weak, at least if absolute accuracy is not an issue but the primary goal is to determine the spectral slope in surface reflectance between the closely spaced MERIS bands. The surface reflectance retrieval over land can thus be performed using climatological mean values of aerosol properties (e.g. continental aerosol located in boundary layer, $\mathrm{AOT}_{900 \mathrm{~nm}}=0.15$ ) in case there is no additional information available.

Above the dark ocean the situation is different, since there is no spectral dependency of the surface reflectance, however, the influence of atmospheric scattering on the measured band ratio is much more pronounced. Here, the surface reflectance can be simulated using the wind speed for parametrizing the sea surface roughness (Cox and Munk, 1954; Koepke, 1984).

Finally, in order to account for the influence of atmospheric scattering, the scattering correction factor $f$ (see Eq. 2) is extracted from tabulated radiative transfer simulations, using radiative transfer code MOMO. As detailed in Sect. $2, f$ specifies the relation between the band ratios in a non-scattering and a scattering atmosphere, as a function of the observing geometry, the TCWV, the surface reflectance and the aerosol loading. By applying the scattering correction factor to the modeled transmittance, the bias in retrieved TCWV due to scattering is eliminated. However, due to uncertainties in auxiliary parameters such as the surface reflectance and the prevailing aerosol type, optical thickness and vertical distribution, a residual uncertainty remains. Due to the significantly stronger influence of scattering above dark surfaces, the resulting uncertainty of the derived water vapour column is much higher above the ocean. Over dark ocean areas (TOA reflectance $<0.1$ ), the true ocean surface reflectance is calculated from the wind speed and the observing geometry (Cox and Munk, 1954; Koepke, 1984). The difference between TOA reflectance and calculated ocean surface reflectance is then used to estimate the aerosol loading and hereupon calculate the scattering correction factor $f$. This procedure is neither possible nor necessary when the sensor is directed towards the direct glitter of the sun, where the influence of the scattering atmosphere is weak and a climatological value of aerosol optical thickness can be used for the calculation of $f$.

A flow chart of the forward modelling operator is shown in Fig. 6.

\subsection{Inversion technique}

In the retrieval scheme the first guess of TCWV is obtained from a simple regression, relating TCWV to a third order polynomial of $\ln (R)$, where the regression coefficients were determined using radiative transfer simulations with MOMO. In the iterative optimization routine, there is a single variable to be fitted, the total columnar water vapour, using a single piece of information, the MERIS band ratio $R$, with a monotonic relation between both figures. The use of background information about TCWV such as e.g. NWP reanalysis data is therefore not necessary over sufficiently bright targets, as it only dilutes the high sensitivity inherent to the MERIS measurement. The straightforward optimization of TCWV is performed by the secant method. It was preferred to the Newton method in spite of its slightly slower convergence, because there is no need to calculate the derivative of the cost function at every retrieval step, so its implementation provides a faster processing in practice.

Starting with the first guess, TCWV is adjusted until the cost function, simply represented by the absolute difference between simulated and measured ratio of normalized radiances in MERIS bands 15 and 14, is below a pre-defined threshold, determined by e.g. the sensor noise.

\subsection{Uncertainty estimate}

Once the iteration procedure has converged the retrieval uncertainty is calculated, taking into account direct measurement errors, that is instrumental noise, as well as the uncertainties of all those parameters that are not part of the state vector but are fixed a priori. These are the surface albedo 

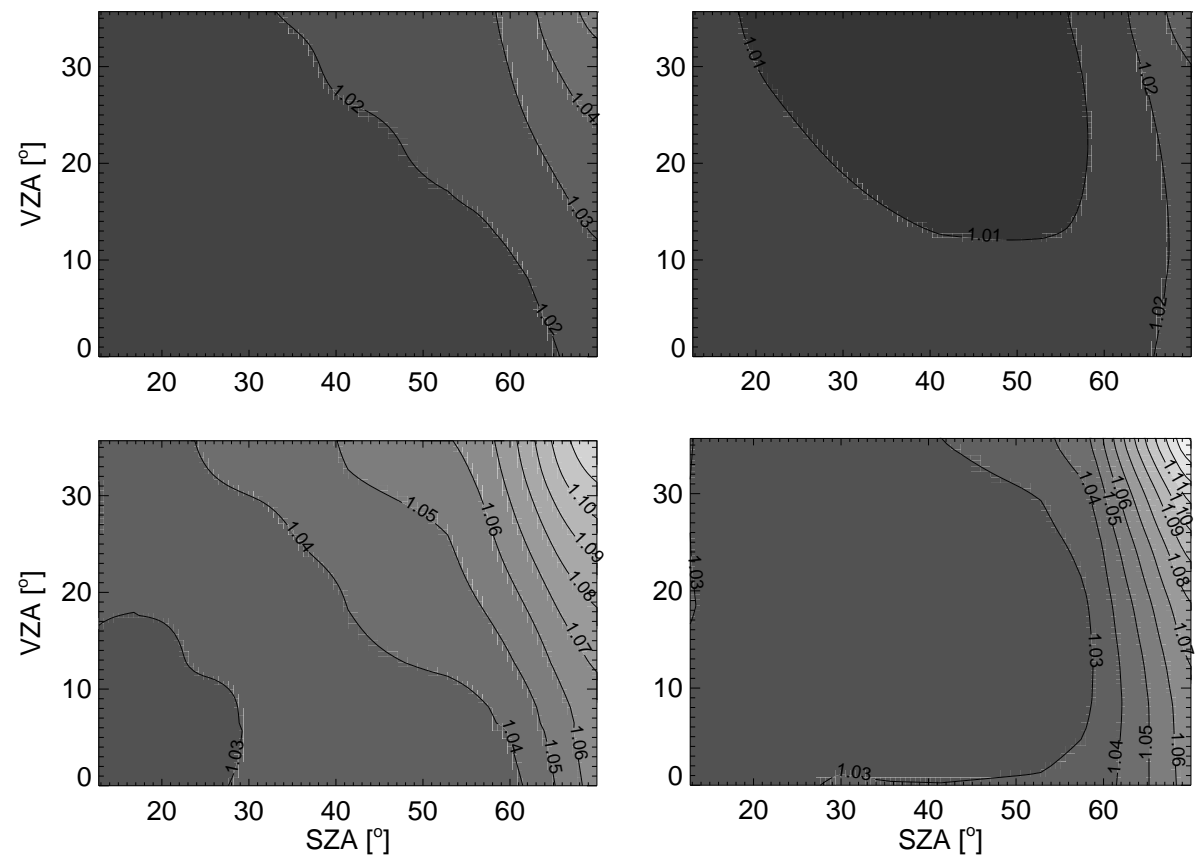

Fig. 5. Scattering correction factor $f$ as a function of viewing and illumination geometry for relative azimuth angle of $180^{\circ}$ (left panels) and $0^{\circ}$ (right panels), with $0^{\circ}$ representing cases where the sensor is placed opposite of the sun, shown for aerosol optical thickness of 0.28 , a surface albedo of 0.4 , TCWV of $56 \mathrm{~mm}$ and a continental aerosol located in the boundary layer (upper panels) and the upper troposphere (lower panels), respectively.

and its spectral dependency, the temperature profile, the surface pressure, the aerosol optical thickness and the aerosol height. The uncertainty introduced by these modelling parameters $b_{i}$ is determined by converting the individual error contributions into measurement space via the modelling parameter Jacobian $K_{b}$ and adding them to the measurement error covariance matrix $\mathbf{S}_{\epsilon}$, which in our case is just a scalar, as we are only using one piece of information, namely the band ratio $R$ :

$\mathbf{S}_{y}=\mathbf{S}_{\epsilon}+K_{b}^{T} S_{b} K_{b}$.

The resulting error covariance matrix $\mathbf{S}_{y}$, again in our case just a scalar variance of $R$ due to all measurement and model parameter uncertainties, is converted into parameter space using the Jacobian $K$, that is the partial derivative of the band ratio $R$ with respect to TCWV at the retrieved state. As a result we get $\hat{S}$, the variance of the retrieved state, that is directly used to define the measurement uncertainty (Rodgers, 2000):

$\hat{S}=\left(K^{T} \mathbf{S}_{y}^{-1} K\right)^{-1}$.

In particular, the contributions of the individual error sources to the uncertainty of the retrieved state are estimated as follows. As detailed in Sect. 3.1, among the above parameters there are 3 candidates affecting the scattering correction factor $f$, namely the absolute surface reflectance, the aerosol optical thickness and the aerosol height. For each of these parameters, an $f_{i}^{*}$ (with $i=1,2,3$ ) is calculated from the look-up tables, by perturbing the input accordingly. The perturbation value is 0.1 in case of aerosol optical thickness and 0.02 for the surface albedo. Since there is no information about the vertical distribution of the aerosols, a large error is assumed by shifting the aerosol layer to the upper troposphere instead of locating it in the boundary layer. Instead of $f$, the $f_{i}^{*}$ are used to correct the simulated transmittance for scattering, in each case resulting in a perturbed band ratio $R_{i}^{*}$. We obtain the deviation of the modeled band ratio $\Delta R_{i}=R_{i}-R$ for each of the three parameters. The $\Delta R_{i}$ are squared and added to the measurement error variance $\mathbf{S}_{\epsilon}$.

In case of surface pressure and temperature, the forward model is used directly to simulate perturbed transmittances that are corrected with an unperturbed scattering correction factor $f$. The assumed uncertainties are $10 \mathrm{hPa}$ for surface pressure and $5 \mathrm{~K}$ for the surface temperature, resulting again in values of $\left(\Delta R_{i}\right)^{2}$ that are added to $\mathbf{S}_{\epsilon}$.

Above land, the uncertainty introduced by the spectral albedo slope is parametrized with the normalized differenced vegetation index of the observed scene, calculated from the reflectance in the visible and near infrared: NDVI $=\left(\rho_{860 \mathrm{~nm}}-\rho_{660 \mathrm{~nm}}\right) /\left(\rho_{860 \mathrm{~nm}}+\rho_{660 \mathrm{~nm}}\right)$. As shown in Fig. 7, the linear extrapolation of the spectral surface albedo $\alpha_{\lambda}$ at $900 \mathrm{~nm}$ from the values at $865 \mathrm{~nm}$ and $885 \mathrm{~nm}$ is a good approximation for vegetated surfaces with an NDVI 


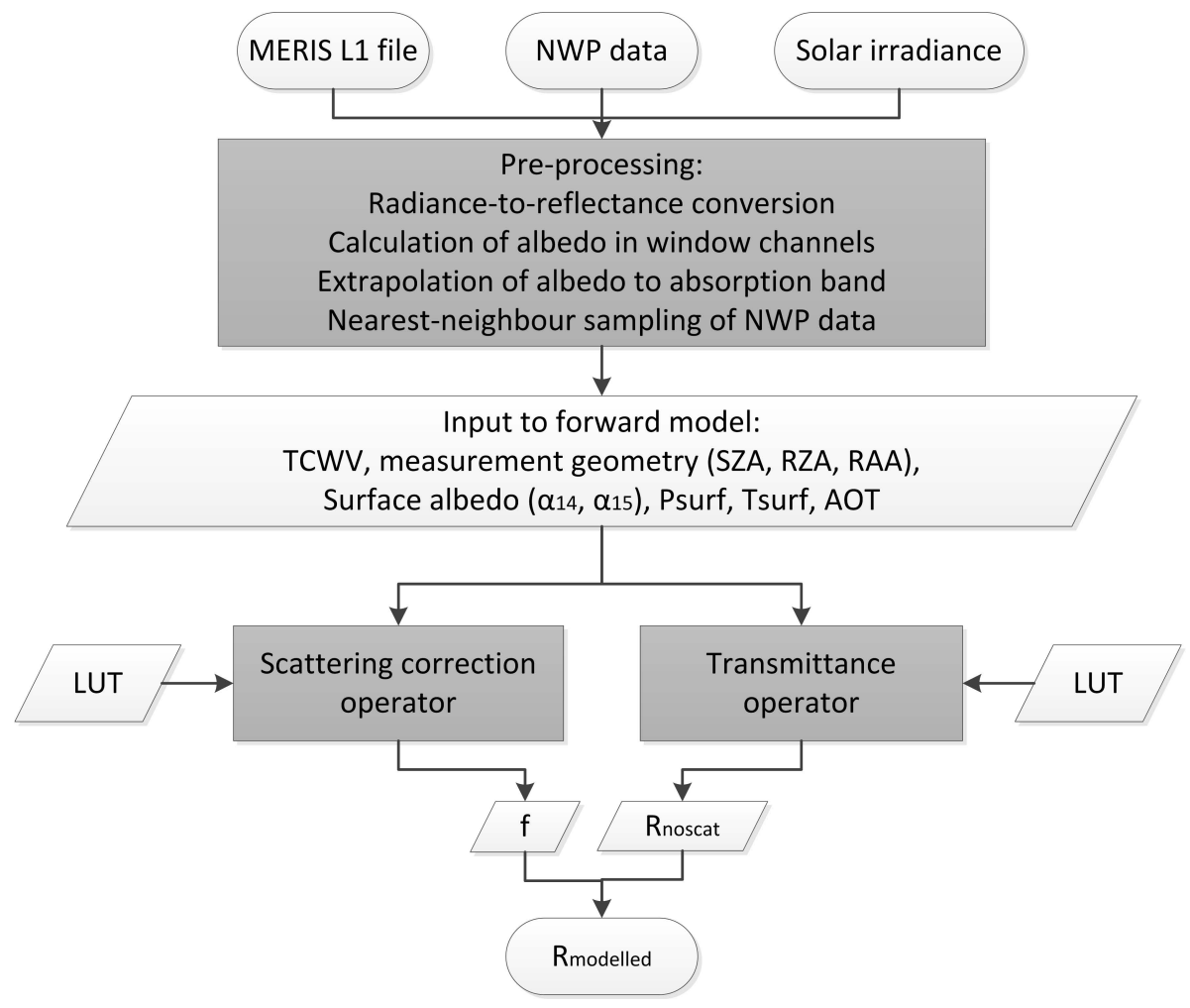

Fig. 6. Flow chart of forward modelling process.

around 0.6 and less accurate for e.g. bare soils or snow covered areas. Therefore, the NDVI is used to estimate the uncertainty of $\alpha_{900 \mathrm{~nm}}$, ranging from 0.001 to 0.003 for snowfree areas up to 0.006 above snow and ice. Similar to the approach detailed above, a perturbed transmittance is calculated from the perturbed albedo slope using the forward model, with the resulting deviation contributing to $\mathbf{S}_{\epsilon}$. As there is no spectral albedo slope above ocean, no error contribution is calculated here.

The final contribution to $\mathbf{S}_{\epsilon}$ is the sensor noise with an assumed signal-to-noise-ratio of 250 .

The resulting overall uncertainty of the retrieved TCWV can exceed $50 \%$ over the dark ocean, where the unknown aerosol vertical distribution and optical thickness is the predominant contributor to the retrieval uncertainty. Over nonvegetated land areas and especially ice and snow-covered regions, the spectral albedo slope uncertainty is an important error contribution. Overall, the uncertainty is typically below $5 \%$ over bright targets.

\section{Validation}

The MERIS TCWV retrieval was thoroughly validated by comparing it to ground-based reference data over land and satellite-borne microwave retrievals from SSM/I and ENVISAT MWR over ocean. The details of these validation

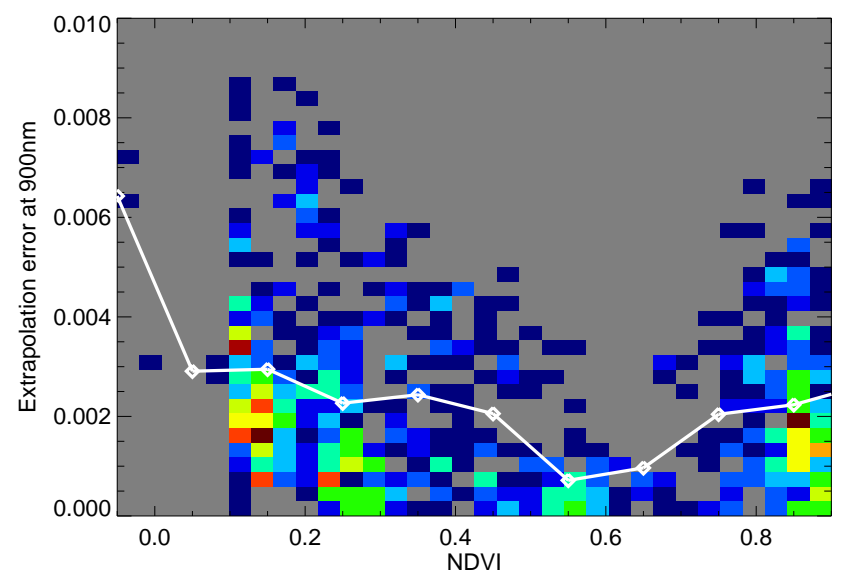

Fig. 7. Normalized frequency of distribution of error of linearly extrapolated surface albedo at $900 \mathrm{~nm}$ (color coded) and resulting error parametrization (white curve) as a function of NDVI.

studies are outlined in Sects. 4.1 and 4.2. Over land, other than a generally high precision, a systematic wet bias of up to $10 \%$ was observed when comparing MERIS TCWV to the various validation data sets. Provided that there is no dry bias in the used validation data, a potential underestimation of the absorption by water vapour around $900 \mathrm{~nm}$ was identified as a possible source of this bias. The water vapour absorption 

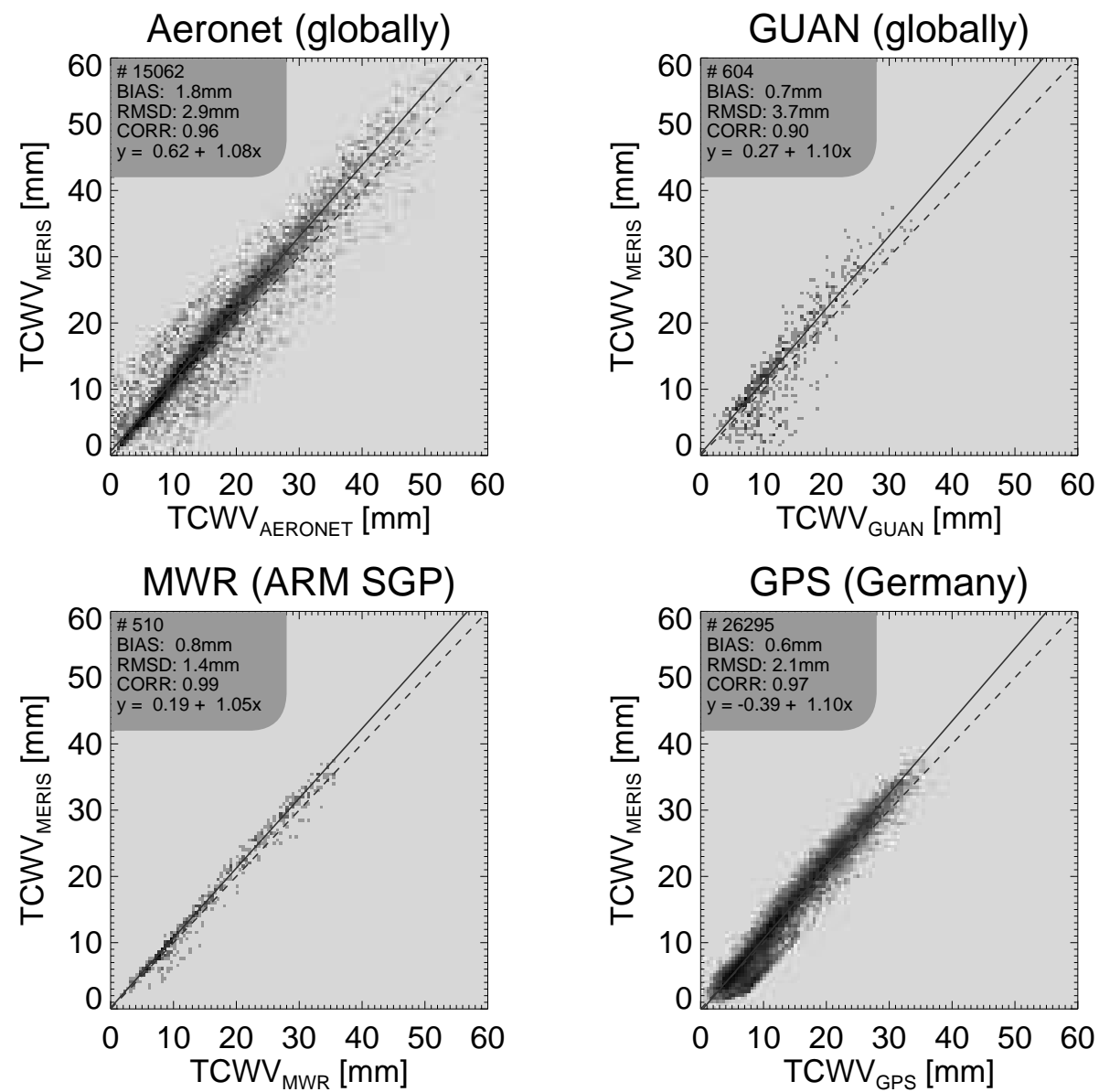

Fig. 8. Normalized frequencies of occurrence for comparisons of MERIS-derived TCWV against AERONET sun-photometer measurements (top left panel), GUAN radiosondes (top right panel), ARM Southern Great Plains microwave radiometer data (bottom left panel) and ground based GPS data (bottom right panel), each for the period 2003-2005. See Fig. 9 for geographic distribution of validation data and text for detailed discussion.

is usually described as a sum of absorption lines, that are cut off at a line-width-dependent spectral distance from the line centre, and an underlying, spectrally slowly varying continuum absorption. However, the strength of the continuum absorption and the optimal line wing cut-off are still under discussion, resulting in an uncertain water vapour optical depth. The observed bias in MERIS TCWV is well within this uncertainty, as demonstrated in Sect. 4.3.

Due to fact that the behaviour of the water vapour continuum is not yet well understood, all validation results shown in Sects. 4.1 and 4.2 are solely based on HITRAN 2008 absorption line parameters (Rothman et al., 2009), hereby neglecting continuum absorption. In order to compensate for not considering continuum absorption, the line wings were not cut off.

\subsection{Validation over land}

The MERIS TCWV retrieval was thoroughly validated over land against various sources of reference data, namely
GUAN radiosonde data, AERONET sun-photometer measurements, ARM microwave radiometer observations and ground-based GPS water vapour monitoring data. For each of these 4 reference data sets, 3 years of data in the period 2003-2005 were compared to the MERIS retrieval. The results for each data set are shown in Fig. 8 as normalized frequency-of-occurrence plots. In each plot, the relative frequency of occurrence is shown in grey shading with high occurrences shown in dark grey, and low occurrences shown in light grey. In the top left corner of each panel the sample size, bias, root mean square deviation (in the following rmsd), the correlation coefficient and the offset and slope of the linear best fit are given.

Each validation data set was filtered for outliers and disturbing influences such as high aerosol loading or cloud contamination. The individual filter criteria are detailed in the according subsections. For the comparison of the satellite observations and the particular ground-based reference data sets, MERIS measurements were spatially averaged in order to account for e.g. the radiosonde displacement, the time gap 
between satellite overpass and ground-based measurement, and the limited accuracy of the MERIS geolocation. Here the value $\mathrm{TCWV}_{i}$ of each considered pixel was weighted with its associated uncertainty $\sigma_{i}^{2}: \mathrm{TCWV}=\sum_{i=0}^{n} w_{i} \cdot \mathrm{TCWV}_{i} / \sum_{i=0}^{n} w_{i}$, with $w_{i}=\frac{1}{\sigma_{i}^{2}}$.

\subsubsection{Validation against Aeronet sun photometer measurements (2003-2005)}

The MERIS TCWV retrieval was compared to a 3-year global set of Aeronet measurements (see Fig. 9 for Aeronet site locations and top left panel in Fig. 8 for validation results). Total precipitable water at the Aeronet stations is derived from sun photometer measurements in the $0.94 \mu \mathrm{m}$ region (Direct Sun Algorithm version 2; Reagan et al., 1987; Schmid et al., 1996) with an estimated accuracy of better than $2 \mathrm{~mm}$ (Michalsky et al., 1995). The Aeronet data was obtained via http://aeronet.gsfc.nasa.gov. In order to account for the differing viewing geometries, the time gap between the Aeronet and the MERIS measurements (constrained to $\leq 30 \mathrm{~min}$ ), and the limited accuracy of the MERIS geolocation, the MERIS measurements were averaged within $10 \mathrm{~km}$ $\times 10 \mathrm{~km}$, centered around the Aeronet station. Each contributing MERIS pixel was weighted with its associated uncertainty. As an additional constraint, the measurements were rejected in case there were less than $20 \%$ of valid MERIS pixels, in order to reduce the fraction of cloud contaminated samples. The filtered subset was additionally screened for high aerosol loadings $($ AOT $>1)$ and outliers, deviating by more than $3 \sigma$.

The comparison of Aeronet and MERIS reveals a wet bias of MERIS of $1.8 \mathrm{~mm}$ and a bias-corrected rmsd of $2.9 \mathrm{~mm}$ between both data sets. The linear fit has a slope of 1.08 and an offset close to 0 , meaning that on average MERIS TCWV is $8 \%$ larger than Aeronet TCWV. Since a wet bias of MERIS was found in all validation studies, it might indicate an underestimation of the water vapour absorption in the spectroscopic database.

\subsubsection{Validation against GUAN radiosonde data (2003-2005)}

A 3-year global set of GUAN (GCOS Upper Air Network) radiosonde data, distributed via the GTS network and extracted from the DWD archive, was used to compare with the MERIS TCWV retrieval (see Fig. 9 for GUAN site locations and top right panel in Fig. 8 for validation results). In order to account for the displacement of the radiosonde during its ascent, the time gap between the GUAN and the MERIS measurements (constrained to $\leq 30 \mathrm{~min}$ ), and the limited accuracy of the MERIS geolocation, the MERIS measurements were averaged within $40 \mathrm{~km} \times 40 \mathrm{~km}$, centered around the GUAN station. Each contributing MERIS pixel was weighted with its associated uncertainty. Cases with less than

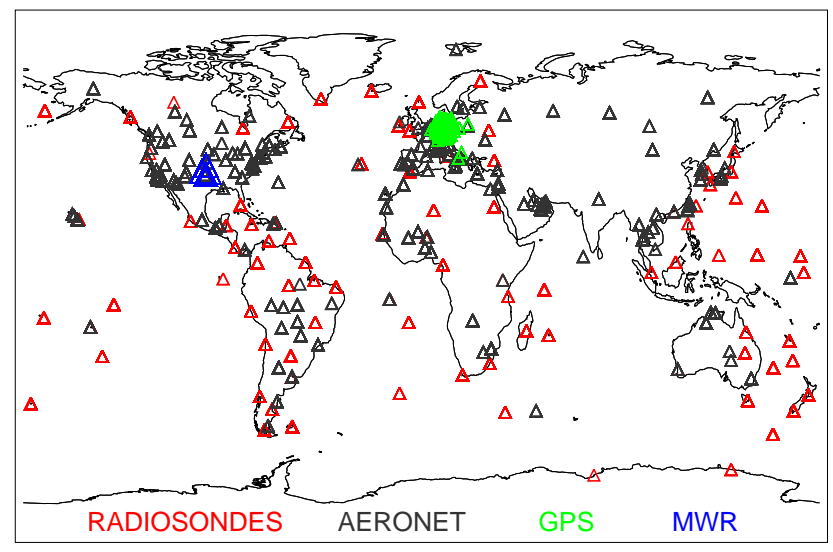

Fig. 9. Geographic distribution of used validation data over land.

$20 \%$ of valid MERIS pixels were rejected in order to reduce the fraction of cloud contaminated samples.

In the subset, filtered for outliers (deviation $\geq 3 \sigma$ ), a bias of $0.7 \mathrm{~mm}$ and a bias-corrected rmsd of $3.7 \mathrm{~mm}$ are found. These values are within the range of the radiosonde uncertainty (Turner et al., 2003; Miloshevich et al., 2004). The linear fit shows a slope of 1.1 and an offset of $0.27 \mathrm{~mm}$.

\subsubsection{Validation against ARM microwave radiometer data (2003-2005)}

A 3-year set of ground-based ARM Southern Great Plains (SGP) microwave radiometer (MWR, software version 4.13) data was used to compare with the MERIS TCWV retrieval (see Fig. 9 for ARM SGP site location and bottom left panel in Fig. 8 for validation results). The MWR instruments are microwave radiometers designed to measure the radiation emitted by atmospheric water vapour and liquid water at frequencies of $23.8 \mathrm{GHz}$ and $31.4 \mathrm{GHz}$ (Turner et al., 2007). Since there is no uncertainty introduced by the background emission of the cold space, ground-based microwave data is considered as one of the most accurate methods for the determination of the water vapour column amount. The measurement uncertainty is expected to be in the range of $0.3 \mathrm{~mm}$ (Turner et al., 2003).

For the comparison, the cloud-free and valid MERIS pixels were averaged within $10 \mathrm{~km} \times 10 \mathrm{~km}$ around the ARM sites. The MWR measurements, provided minute-by-minute, were averaged in a \pm 15 min time frame around the ENVISAT overpass. This averaging was performed in order to minimize disturbing effects such as undetected clouds, inaccurate MERIS geolocation and the different observing geometries.

The comparison of MERIS and MWR TCWV values shows an almost perfect agreement with a wet bias of $0.8 \mathrm{~mm}$ and a root mean square deviation of merely $1.4 \mathrm{~mm}$. Apart from the fact that the comparison is limited to the ARM SGP site and is therefore not representative for 
global observations, this result indicates that the accuracy of MERIS TCWV over reasonably bright land surfaces is outstanding. The wet bias and the linear fit slope of 1.05 indicate again, that the gaseous absorption of water vapour was potentially underestimated.

\subsubsection{Validation against ground-based GPS data (2003-2005)}

Finally, a 3-year data set of GPS water vapour monitoring data was used to compare with the MERIS TCWV retrieval. The analysis of the temperature- and humiditydependent delay of the GPS signal emitted by satellites and received by ground stations allows the retrieval of TCWV (Bender and Raabe, 2007) with an accuracy in the range of 1-2 mm (Gendt et al., 2004). The GPS-data was provided by GFZ Potdsam and was derived from 153 ground based GPS stations in Central Europe using the EPOS.P.V2 software (Gendt et al., 1999, see Fig. 9 for GPS site locations and bottom right panel in Fig. 8 for validation results).

Again, MERIS measurements were averaged over $10 \mathrm{~km} \times 10 \mathrm{~km}$ to account for temporal gaps and the differences in observing geometries. Cases with less than $20 \%$ of valid MERIS pixels were rejected in order to reduce the fraction of cloud contaminated samples. A relatively large portion of points, sitting in the lower left part of the plot and indicating an underestimation of TCWV by MERIS in dry conditions, is due to failing cloud detection over snow covered parts of Germany in winter. After filtering out these cases, a bias of $0.6 \mathrm{~mm}$ and a rmsd of $2.1 \mathrm{~mm}$ results. In accordance with the results of the comparisons against the other reference data sets, the linear fit has a slope of 1.1 and an offset of $-0.39 \mathrm{~mm}$.

\subsection{Validation over ocean}

\subsubsection{Validation against SSM/I microwave data}

The Special Sensor Microwave/Imager (SSM/I) is a passive, seven-channel, four-frequency microwave radiometer which is operated on the DMSP (Unites States Air Force Defense Meteorological Satellite Program) satellite series. Depending on the channel, SSM/I has a footprint of several tens of kilometers. Due to the well known emissivity of the water surface and the high radiometric accuracy of the instrument $(0.4-0.7 \mathrm{~K}), \mathrm{SSM} / \mathrm{I}$-derived TCWV is regarded as an accurate retrieval source over ocean. The used SSM/I TCWV data was retrieved in the frame of the ESA GlobVapour project with an algorithm based on the work by Phalippou (1996) and Deblonde (2001). In a comparison against MWR measurements at the Nauru ARM site, an rmse of $1.5 \mathrm{~mm}$ and a bias of $-0.5 \mathrm{~mm}$ were found (M. Stengel, Deutscher Wetterdienst, personal communication, 2011).

The data used for the comparison stems from the F13 and F14 satellites with a local equator crossing time in the early morning. Both the time gap of $4-5 \mathrm{~h}$ between SSM/I and MERIS observations and the fact that microwave observations of TCWV are possible under all sky conditions whereas MERIS provides only cloud-free measurements have to be taken into account for the interpretation of the results.

In order to account for the differences in footprint and overpass time, both data sets were averaged within $0.5^{\circ} \times 0.5^{\circ}$ boxes. Cases with less than $30 \%$ of valid MERIS pixels within a box were excluded from the analysis. The accuracy of the MERIS water vapour retrieval is expected to depend on the reflectance of the ocean surface, as over the dark ocean the signal is dominated by the aerosol height and optical depth whereas the unknown impact of atmospheric scattering is less important over bright targets. Due to the forenoon orbit of ENVISAT and the observing geometry of MERIS, direct reflection of the incoming sun light occurs in the eastern part of the MERIS swath, whereas the central and western parts of the MERIS swath are mainly covered by dark ocean. The left panel of Fig. 10 shows the relative frequency of occurrence for the comparison of MERIS and $\mathrm{SSM} / \mathrm{I}$ derived TCWV in the $0.5^{\circ} \times 0.5^{\circ}$ boxes. The background, grey shaded distribution represents a moderately filtered data volume (20\% valid MERIS pixels and standard deviation less than $5 \mathrm{~mm}$ in $0.5^{\circ} \times 0.5^{\circ}$ box) whereas the foreground, color coded distribution represents a more strictly filtered data volume over the brighter parts of the ocean $(20 \%$ valid MERIS pixels and standard deviation less than $2 \mathrm{~mm}$ in $0.5^{\circ} \times 0.5^{\circ}$ box, cloudy cases excluded, ocean reflectance $\rho \geq 0.08$ ). The root mean square deviation between TCWV $\mathrm{TERIS}_{\text {and }} \mathrm{TCWV}_{\mathrm{SSM} / \mathrm{I}}$ is $8.6 \mathrm{~mm}$ for the moderately filtered cases and drops to $5.2 \mathrm{~mm}$ for the stricter filtering. The bias of $-0.3 \mathrm{~mm}$, influenced by undetected clouds, is modified to $2.5 \mathrm{~mm}$, accordingly. Although there is a time gap of 4-5 h between both measurements and the foot prints and measurement techniques differ, the relatively large bias hints at deficiencies of the MERIS retrieval. Apart from the potential underestimation of optical depth for a given water vapour column amount, a possible source for the strong wet bias could be the scattering correction factor, since it was simulated for a Lambertian surface, which is a good approximation for most land surfaces but not applicable over ocean close to sun glint.

\subsubsection{Validation against ENVISAT MWR data}

The ENVISAT microwave radiometer (MWR) is a passive, dual-channel, nadir-pointing radiometer, operating at frequencies of $23.8 \mathrm{GHz}$ and $36.5 \mathrm{GHz}$. Its primary objective is to support the tropospheric path correction of the ENVISAT radar altimeter signal by measurements of the atmospheric humidity over ocean. The data used for the comparison was provided by CLS, France, and generated using an algorithm of Obligis et al. (2006). The MWR has a $20 \mathrm{~km} \times 20 \mathrm{~km}$ footprint at nadir, so only the central part of the MERIS swath can be compared to MWR data. As sun glint is found mainly 

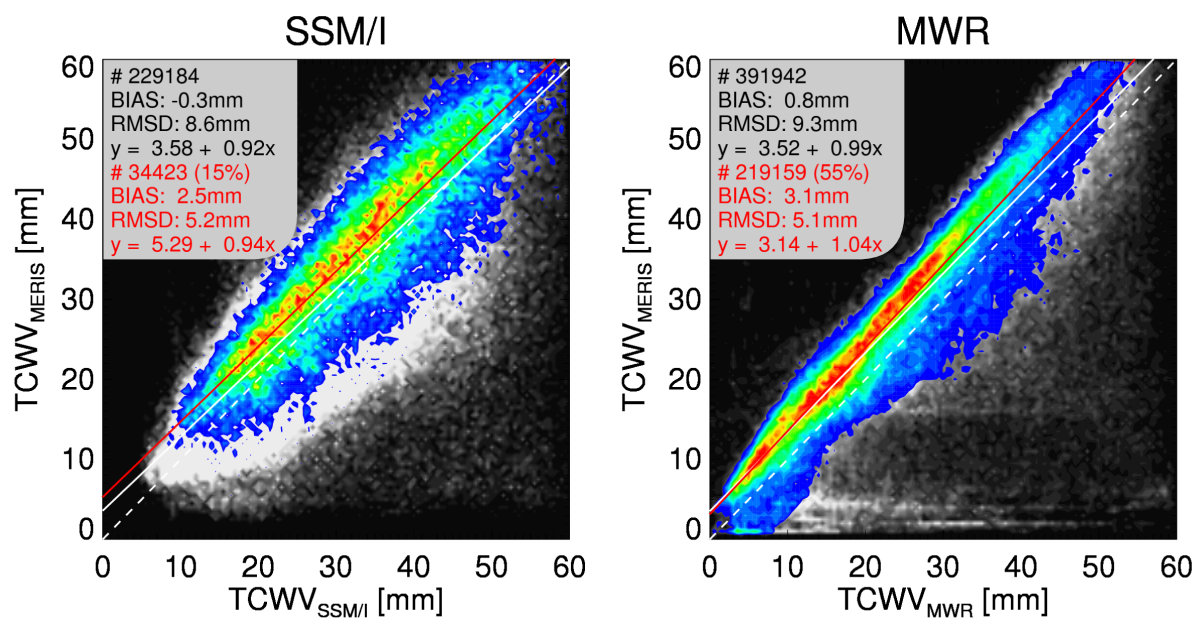

Fig. 10. Left panel: normalized frequency of occurrence for global comparison of SSM/I and MERIS TCWV over ocean (July 2007). Both retrievals were averaged within $0.5^{\circ} \times 0.5^{\circ}$ degree boxes in order to account for the differing footprints and different observation times. Right panel: Normalized frequency of occurrence for direct, global comparison of ENVISAT MWR and MERIS TCWV over ocean (OctoberDecember 2002, June-September 2007). MERIS measurements were averaged within $20 \times 20 \mathrm{~km}^{2}$ in order to match MWR footprint.

Gray shaded background indicates distribution of compared cases without filtering, with the black text specifying statistical analysis and white solid line representing the linear fit. Color coded foreground indicates the distribution of cases, filtered for undetected clouds (lower right regions), high MERIS uncertainty and dark ocean reflectance ( $\rho \geq 0.08$, applied to SSM/I comparison only). See text for more detailed description.

in the eastern part of the MERIS swath, the comparison is limited to the darker parts of the ocean, where MERIS is expected to perform less accurately. As MWR provides only two channels, its accuracy is expected to be reduced as compared to SSM/I data. Since there is no time gap between MWR and MERIS observations.

Figure 10 (right panel) shows the direct comparison between MWR and MERIS TCWV for four months (October and November 2002 and June and July 2007) of global observations over ocean. MERIS measurements were averaged over $20 \mathrm{~km} \times 20 \mathrm{~km}$ in order to match the MWR footprint and excluded in case less than $20 \%$ of the MERIS pixels were valid within this box. The scatter plot shows the relative frequency of occurrence of all resulting cases in grey shading, overplotted with a subset of cases coded in color. The cases occurring in the lower right corner of the diagram and those $20 \mathrm{~km} \times 20 \mathrm{~km}$ boxes with a high standard deviation of MERIS TCWV were filtered out for the subset in order exclude cloudy cases that were falsely classified as clear sky by the MERIS cloud mask. The analysis of the filtered data revealed a bias of $3.1 \mathrm{~mm}$ between MERIS and MWR with a bias-corrected root mean square deviation of $5.1 \mathrm{~mm}$. This result is in accordance with the validation of MERIS TCWV against SSM/I observations.

\subsection{Sensitivity to water vapour continuum absorption}

The spectral structure and strength of the absorption of solar radiation by atmospheric water vapour is commonly described by the Voigt line profile, defining the dependence of the line strength, shape and width as a function of pressure and temperature. The Voigt profile is known to accurately describe the absorption close to the line centres, but to result in a suboptimal description of the far wings of the lines. It is therefore common to cut off the line wings at a certain spectral distance from the line centres and describe the residual absorption and other spectrally slowly varying effects, such as the absorption due to water vapour dimers, by a water vapour continuum model. Although the water vapour continuum is much smaller in the NIR as compared to the thermal regions, it still has a significant influence on the resulting total gaseous optical depth. In this work, the widely used LBLRTM code (Clough et al., 2005) was used to calculate the absorption by water vapour, embodying the MTCKD model for the description of the continuum absorption. However, recent publications such as the paper by Ptashnik et al. (2011), indicate that the strength of the continuum absorption is still highly uncertain, giving rise to the question whether the systematic wet bias observed in the validation of the MERIS TCWV retrieval is caused by an underestimation of the water vapour absorption.

Figure 11 shows the effect of adding the continuum absorption on the spectral structure of the gaseous optical depth in a spectral window between $895 \mathrm{~nm}$ and $905 \mathrm{~nm}$ for four different setups: 

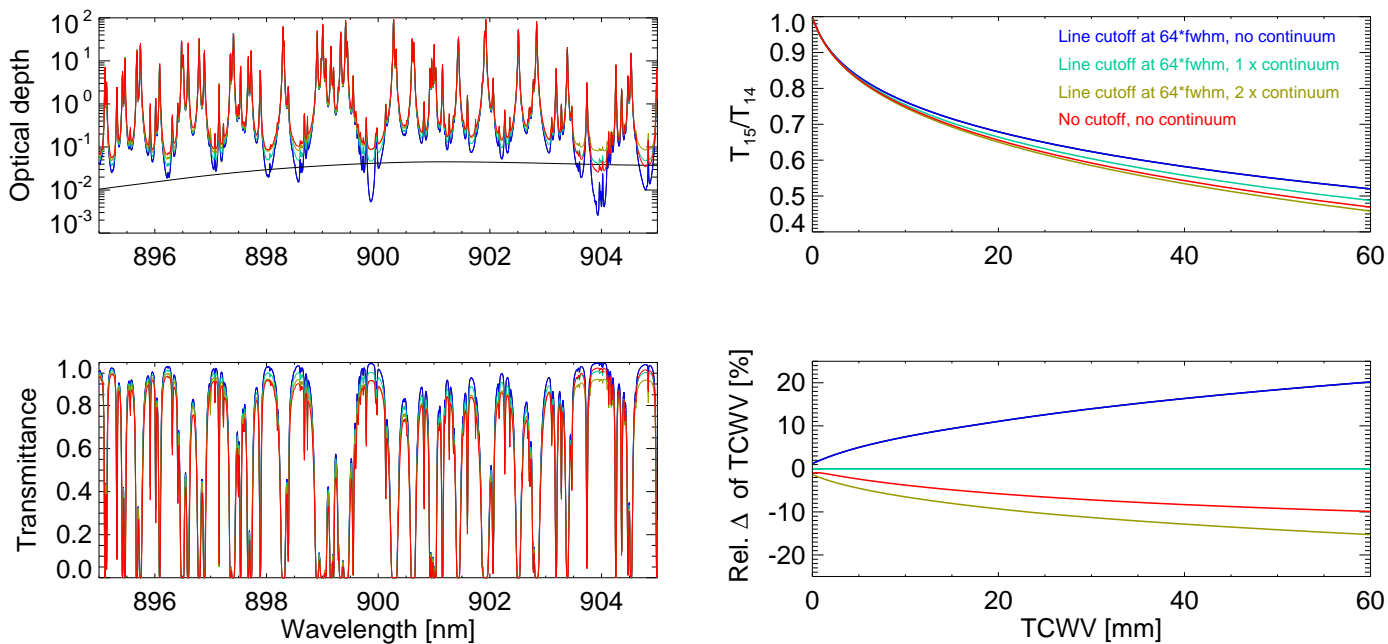

Fig. 11. Left panels: sensitivity of optical depth (upper figure) and transmittance (lower figure) at $900 \mathrm{~nm}$ to spectroscopic assumptions. The colors of the curves denote the underlying line cutoff width and continuum absorption, as indicated in the upper right plot. The continuum optical depth is shown in black in the upper left panel. Right panels: Transmittance ratio of MERIS bands 15 and 14 as a function of TCWV (upper) and corresponding relative difference of derived TCWV, in relation to retrieval based on line cutoff at $64 \cdot \mathrm{fwhm}$ and a MT-CKD continuum.

All results shown were calculated for a mid-latitude summer atmosphere, a surface pressure of $1050 \mathrm{hPa}$ and an air-mass of 3 .

1. Line wings are cut off at a spectral distance of 64 times the full width at half maximum (fwhm) of each line and no continuum optical depth is added.

2. As for setup 1, but a MT-CKD continuum is added.

3. As for setup 1, but a doubled MT-CKD continuum is added.

4. The line wings are not cut off and no continuum is added.

The lower right plot shows the equivalent change of TCWV for the four different setups, relative to the common approach (setup 2). If the continuum is neglected (setup 1), an overestimation of up to $20 \%$ of TCWV results. Accordingly, adding a doubled continuum results in an underestimation of similar magnitude. Note that the relative overestimation is stronger for high values of TCWV, meaning that the continuum has a stronger effect in case of humid atmospheres. When the line wing cutoff is not performed and no continuum is added, the resulting optical depth is higher than for the common approach, but lower than adding a doubled continuum. Note that setups 1 (no continuum), 2 (including continuum) and 4 (no continuum, no cutoff) result in a wet bias of MERIS TCWV, whereas setup 3 (doubled continuum absorption) results in an almost perfect fit against ground-based MWR observations.

The observed wet bias of MERIS TCWV is in agreement with validation results obtained by Gao and Kaufman (2003) for near infrared measurements of the Moderate Resolution Imaging Spectroradiometer.

\section{Summary and outlook}

We present a new algorithm for the retrieval of TCWV from MERIS measurements in the rsvt-absorption band in the near infrared spectral range. It is based on fast forward simulation of the absorption by water vapour, corrected for the difference in surface albedo between the used MERIS bands and the impact of atmospheric scattering on the measured band ratio. The influence of the temperature- and pressure-profile on the water vapour absorption lines is taken into account. The algorithm provides realistic uncertainty estimates on a pixel-by-pixel basis, considering all relevant error sources of uncertainty, including instrumental noise as well as forward model parameter uncertainties. The retrieved data is thus well qualified for the generation of level 3 products such as global time series.

Over land, an extensive validation study against various sources of ground based reference data sets generally revealed a high accuracy of MERIS TCWV with biascorrected root mean square deviations of $2.9 \mathrm{~mm}$ (Aeronet sun photometer measurements), $3.7 \mathrm{~mm}$ GUAN radiosondes, $1.4 \mathrm{~mm}$ (ARM MWR measurements) and $2.1 \mathrm{~mm}$ (German ground-based GPS data). In all comparisons a wet bias of MERIS TCWV between $0.7 \mathrm{~mm}$ (GPS, GUAN, ARM MWR) and $1.8 \mathrm{~mm}$ (Aeronet) was found. A potentially improper description of the water vapour continuum absorption is a possible explanation for this wet bias, especially in view of the fact that similar effects have been observed for MODIS observations in the near infrared spectral range (Gao and Kaufman, 2003). It was shown that the MERIS bias is within the range of this spectroscopic 
uncertainty. A different, possible explanation for the observed wet bias could be the instrument calibration, such as e.g. over-corrected instrumental stray light, as observed in the MERIS oxygen A band channel for some parts of the field of view (Lindstrot et al., 2010).

The MERIS TCWV retrieval was validated over ocean using coincident ENVISAT MWR data and SSM/I observations. Both comparisons showed a wet bias of $2-3 \mathrm{~mm}$, hinting at deficiencies in the description of the radiative interaction between atmosphere and ocean surface. The biascorrected root mean square deviation between MERIS and MWR and SSM/I is in the range of $5 \mathrm{~mm}$, which is a reasonable value, given the uncertainty of the atmospheric scattering component over ocean.

In the frame of the ESA DUE GlobVapour project, the presented MERIS TCWV algorithm was used above land and coastal waters and combined with SSM/I derived TCWV above open ocean for a generation of a global time series. The Ocean and Land Colour Instrument (OLCI) on-board the Sentinel-3 satellite will continue the MERIS measurements during the next decades.

Acknowledgements. This work was performed in the frame of the ESA DUE GlobVapour project (ESRIN/AO/1-6090/09/I-OL). The ENVISAT MWR data was provided by CLS in the frame of its RA2-MWR ESL L1b activities (ESA contract ENVI-SPPA-EOPGSW-10-0034). The authors would like to thank Bruno Picard of CLS for providing the ENVISAT MWR data and Marc Schröder of Deutscher Wetterdienst for providing SSM/I data. The SSM/I water vapour data has been produced and provided in the frame of the ESA DUE GlobVapour project.

Edited by: A. J. M. Piters

\section{References}

Albert, P., Bennartz, R., Preusker, R., Leinweber, R., and Fischer, J.: Remote Sensing of Atmospheric Water Vapor Using the Moderate Resolution Imaging Spectroradiometer (MODIS), J. Atmos. Ocean. Tech., 22, 309-314, 2005.

Bender, M. and Raabe, A.: Preconditions to ground based GPS water vapour tomography, Ann. Geophys., 25, 1727-1734, doi:10.5194/angeo-25-1727-2007, 2007.

Bennartz, R. and Fischer, J.: A modified $k$-distribution approach applied to narrow band water vapour and oxygen absorption estimates in the near infrared, J. Quant. Spectrosc. Ra., 66, 539-553, 2000.

Bennartz, R. and Fischer, J.: Retrieval of columnar water vapour over land from back-scattered solar radiation using the Medium Resolution Imaging Spectrometer (MERIS), Remote Sens. Environ., 78, 271-280, 2001.

Clough, S. A., Shephard, M. W., Mlawer, E. J., Delamere, J. S., Iacono, M. J., Cady-Pereira, K., Boukabara, S., and Brown, P. D.: Atmospheric radiative transfer modeling: a summary of the AER codes, Short Communication, J. Quant. Spectrosc. Ra., 91, 233$244,2005$.
Cox, C. and Munk, W.: Measurement of the Roughness of the Sea Surface from Photographs of the Sun's Glitter, J. Opt. Soc. Am., 44, 838-850, 1954.

Deblonde, G.: NWP SAF User's Guide: Standalone 1D-var scheme for the SSM/I, SSMIS and AMSU, NWPSAF-MO-UD-001 Version 1.0, 2001.

Delwart, S., Preusker, R., Bourg, L., Santer, R., Ramon, D., and Fischer, J.: MERIS inflight spectral calibration, Int. J. Remote Sens., 28, 479-496, 2007.

Fell, F. and Fischer, J.: Numerical simulation of the light field in the atmosphere-ocean system using the matrix-operator method, J. Quant. Spectrosc. Ra., 3, 351-388, 2001.

Fischer, J.: High-resolution spectroscopy for remote sensing of physical cloud properties and water vapour, in: Current problems in atmospheric radiation, edited by: Lenoble, J. and Geleyn, J.-F., Deepak Publishing, Hampton, Virginia, USA, 151-156, 1988.

Fischer, J. and Grassl, H.: Radiative transfer in an atmosphereocean system: an azimuthally dependent matrix-operator approach, Appl. Optics, 23, 1035-1039, 1984.

Fischer, J., Leinweber, R., and Preusker, R.: ATBD 2.4 Retrieval of Total Water Vapour Content from MERIS Measurements, Algorithm Theoretical Basis Document, European Space Agency, http://envisat.esa.int/instruments/meris/ atbd/atbd_2.4.pdf (last access: 20 March 2012), 2010.

Gao, B.-C. and Kaufman, Y. J.: Water vapor retrievals using Moderate Resolution Imaging Spectroradiometer (MODIS) near-infrared channels, J. Geophys. Res., 108, 4389, doi:10.1029/2002JD003023, 2003.

Gendt, G., Dick, G., and Stihne, W.: GFZ Analysis Center of IGS - Annual Report 1998, IGS 1998 Technical Reports, edited by: Gowey, K., Neilan, R., and Moore, A., Tech. rep., Jet Propulsion Laboratory, Pasadena, 1999.

Gendt, G., Dick, G., Reigber, C., Tomassini, M. Y. L., and Ramatschi, M.: Near Real Time GPS Water Vapor Monitoring for Numerical Weather Prediction in Germany, J. Meteorol. Soc. Jpn., 82, 361-370, 2004.

Guanter, L., Gómez-Chova, L., and Moreno, J.: Coupled retrieval of aerosol optical thickness, columnar water vapor and surface reflectance maps from ENVISAT/MERIS data over land, Remote Sens. Environ., 112, 2898-2913, doi:10.1016/j.rse.2008.02.001, 2008.

Heise, S., Wickert, J., Beyerle, G., Schmidt, T., and Reigber, C.: Global monitoring of tropospheric water vapor with GPS radio occultation aboard CHAMP, Adv. Space Res., 37, 2222-2227, doi:10.1016/j.asr.2005.06.066, 2006.

Hollstein, A. and Fischer, J.: Radiative transfer solutions for coupled atmosphere ocean systems using the matrix operator technique, J. Quant. Spectrosc. Ra., 113, 536-548, doi:10.1016/j.jqsrt.2012.01.010, 2012.

Koepke, P.: Effective reflectance of oceanic whitecaps, Appl. Optics, 23, 1816-1824, 1984.

Lindstrot, R., Preusker, R., and Fischer, J.: The empirical correction of stray light in the MERIS oxygen A band channel, J. Atmos. Ocean. Tech., 27, 1185-1194, 2010.

McClatchey, R., Fenn, R., Selby, J., Volz, F., and Garing, J.: Optical Properties of the Atmosphere, 3rd Edn., Air Force Cambridge Research Laboratories, 1972. 
Michalsky, J. J., Liljegren, J. C., and Harrison, L. C.: A comparison of Sun photometer derivations of total column water vapor and ozone to standard measures of same at the Southern Great Plains Atmospheric Radiation Measurement site, J. Geophys. Res., 100, 25995-26003, doi:10.1029/95JD02706, 1995.

Miloshevich, L. M., Paukkunen, A., Vömel, H., and Oltmans, S. J.: Development and Validation of a Time-Lag Correction for Vaisala Radiosonde Humidity Measurements, J. Atmos. Ocean. Tech., 21, 1305-1327, 2004.

Muller, J.-P., Preusker, R., Fischer, J., Zuhlke, M., Brockmann, C., and Regner, P.: ALBEDOMAP: MERIS land surface albedo retrieval using data fusion with MODIS BRDF and its validation using contemporaneous EO and in situ data products, in: IGARSS International Geoscience and Remote Sensing Symposium 2007, 2404-2407, doi:10.1109/IGARSS.2007.4423326, 2007.

Noël, S., Buchwitz, M., Bovensmann, H., and Burrows, J. P.: Retrieval of total water vapour column amounts from GOME/ERS2 data, Adv. Space Res., 29, 1697-1702, 2002.

Noël, S., Buchwitz, M., and Burrows, J. P.: First retrieval of global water vapour column amounts from SCIAMACHY measurements, Atmos. Chem. Phys., 4, 111-125, doi:10.5194/acp-4111-2004, 2004.

Obligis, E., Eymard, L., Tran, N., Labroue, S., and Femenias, P.: First three years of the microwave radiometer aboard ENVISAT: In-fight calibration, processing, and validation of the geophysical products, J. Atmos. Ocean. Tech., 23, 802-814, 2006.

Phalippou, L.: Variational retrieval of humidity profile, wind speed and cloud liquid-water path with the SSM/I: Potential for numerical weather prediction, Q. J. Roy. Meteorol. Soc., 122, 327-355, 1996.

Pougatchev, N., August, T., Calbet, X., Hultberg, T., Oduleye, O., Schlüssel, P., Stiller, B., Germain, K. St., and Bingham, G.: IASI temperature and water vapor retrievals - error assessment and validation, Atmos. Chem. Phys., 9, 6453-6458, doi:10.5194/acp9-6453-2009, 2009.

Ptashnik, I. V., Shine, K. P., and Vigasin, A. A.: Water vapour self-continuum and water dimers: 1 . Analysis of recent work, J. Quant. Spectrosc. Ra., 112, 1286-1303, 2011.

Rast, M., Bezy, J. L., and Bruzzi, S.: The ESA Medium Resolution Imaging Spectrometer MERIS - A review of the instrument and its mission, Int. J. Remote Sens., 20, 1681-1702, 1999.

Reagan, J. A., Thome, K., Herman, B., and Gali, R.: Water vapor measurements in the $0.94 \mathrm{~m}$ absorption band: Calibration, measurements, and data applications, in: Proceedings: International Geoscience and Remote Sensing Symposium, '87 Symposium, Ann Arbor, Michigan, IEEE, 63-67, 1987.
Rodgers, C.: Inverse Methods for Atmospheric Sounding: Theory and Practice, World Scientific, London, 2000.

Rothman, L., Gordon, I., Barbe, A., Benner, D., Bernath, P., Birk, M., Boudon, V., Brown, L., Campargue, A., Champion, J.-P., Chance, K., Coudert, L., Dana, V., Devi, V., Fally, S., Flaud, J.M., Gamache, R., Goldman, A., Jacquemart, D., Kleiner, I., Lacome, N., Lafferty, W., Mandin, J.-Y., Massie, S., Mikhailenko, S., Miller, C., Moazzen-Ahmadi, N., Naumenko, O., Nikitin, A., Orphal, J., Perevalov, V., Perrin, A., Predoi-Cross, A., Rinsland, C., Rotger, M., Simeckova, M., Smith, M., Sung, K., Tashkun, S., Tennyson, J., Toth, R., Vandaele, A., and Auwera, J. V.: The HITRAN 2008 molecular spectroscopic database, J. Quant. Spectrosc. Ra., 110, 533-572, doi:10.1016/j.jqsrt.2009.02.013, 2009.

Schlüssel, P. and Emery, W. J.: Atmospheric water vapour over oceans from SSM/I measurements, Int. J. Remote Sens., 11, 753766, doi:10.1080/01431169008955055, 1990.

Schmid, B., Thome, K., Demoulin, P., Peter, R., Matzler, C., and Sekler, J.: Comparison of modeled and empirical approaches for retrieving columnar water vapor from solar transmittance measurements in the 0.94 micron region, J. Geophys. Res., 101, 9345-9358, 1996.

Susskind, J., Barnet, C. D., and Blaisdell, J. M.: Retrieval of atmospheric and surface parameters from AIRS/AMSU/HSB data in the presence of clouds, IEEE T. Geosci. Remote, 41, 390-409, 2003.

Thuillier, G., Hersé, M., Labs, D., Foujols, T., Peetermans, W., Gillotay, D., Simon, P. C., and Mandel, H.: The solar spectral irradiance from 200 to $2400 \mathrm{~nm}$ as measured by the SOLSPEC spectrometer from the ATLAS and EURECA missions, Sol. Phys., 224, 1-22, 2003.

Turner, D. D., Lesht, B. M., Clough, S., Liljegren, J., Revercomb, H. E., and Tobin, D. C.: Dry bias and variability in VAISALA RS80-H radiosondes: The ARM experience, J. Atmos. Ocean. Tech., 20, 117-132, 2003.

Turner, D. D., Clough, S. A., Liljegren, J. C., Clothiaux, E. E., Cady-Pereira, K., and Gaustad, K. L.: Retrieving liquid water path and precipitable water vapor from Atmospheric Radiation Measurement (ARM) microwave radiometers, IEEE T. Geosci. Remote, 45, 3680-3690, doi:10.1109/TGRS.2007.903703, 2007.

US GeologicalSurvey: Global 30 Arc-Second Elevation Data Set, http://www1.gsi.go.jp/geowww/globalmap-gsi/gtopo30/ README.html (last access: 20 March 2012), 1996. 